\title{
GENERALIZATION OF STATISTICALLY CONVERGENT
}

\author{
RABİA SAVAŞ AND RICHARD F. PATTERSON
}

\begin{abstract}
In the late 1950's and early 1960's Kurzweil and Henstock presented the concept of Gauge integral. Following their results, Savas and Patterson extended this concept to summability theory by considering $f(\psi)$ real valued function which is integrable in the Gauge sense on $(1, \infty)$. The goal of this paper includes the extension of these notion to statistical convergence. This will be accomplished by presenting the definition of statistically convergent to $L$ via cardinality in Lebesgue sense. Natural implications and variations are also presented.
\end{abstract}

В кінці 1950-х та на початку 1960-х років Курцвайль і Хенсток сформулювали концепцію калібрувального інтеграла. Савас і Паттерсон поширили це на теорію підсумовування, розглянувши дійсні функції $f(\psi)$, інтегровні в калібрувальному сенсі на $(1, \infty)$. Метою цієї роботи є поширення цього поняття на випадок статистичної збіжності. Для цього дається визначення статистичної збіжності за мірою Лебега. Обговорюються наслідки та можливі варіанти цього підходу.

\section{Introduction, Preliminaries and Definitions}

In 1957 Kurzweil [5] presented a new concept of integral which is called Gauge Integral. This notion allows us to extend the class of integrable functions beyond those of Lebesgue integrable. In [4] Henstock refined and placed this notion on a more solid foundation. Let us now present the definition of Gauge integral that was defined in [11].

Definition 1.1. [11] A tagged partition of an interval $I=[a, b]$ is a finite set or ordered pairs

$$
D=\left\{\left(t_{i}, I_{i}\right): 1 \leq i \leq m\right\}
$$

where $\left\{I_{i}: 1 \leq i \leq m\right\}$ is a partition of $I$ consisting of closed non overlapping subintervals and $t_{i}$ is a point belonging to $I_{i} ; t_{i}$ is called the tag associated with $I_{i}$. If $f: I \rightarrow \mathbb{R}$, the Riemann sum of $f$ with respect to $D$ is defined to be

$$
S(f, D)=\sum_{i=1}^{m} f\left(t_{i}\right) \ell\left(I_{i}\right)
$$

where $\ell\left(I_{i}\right)$ is the length of the subinterval $I_{i}$. If $\delta: I \rightarrow(0, \infty)$ is a positive function, we define an open interval valued function on $I$ by setting $\gamma(t)=(t-\delta(t), t+\delta(t))$. If $I_{i}=\left[x_{i}, x_{i+1}\right]$, we can write $t_{i} \in I_{i} \subset \gamma\left(t_{i}\right)$ instead of $t_{i}-\delta<x_{i} \leq t_{i} \leq x_{i+1}<t_{i}+\delta$. Any interval $\gamma$ defined on $I$ such that $\gamma(t)$ is an open interval containing $t$ for each $t \in I$ is called a Gauge on $I$. Let us denote the set of all such interval by $\Delta_{G}$. If $D=\left\{\left(t_{i}, I_{i}\right): 1 \leq i \leq m\right\}$ is a tagged partition of $I$ and $\gamma$ is a Gauge on $I$, we say that $D$ is $\gamma-$ fine if $t_{i} \in I_{i} \subset \gamma\left(t_{i}\right)$ is satisfied. Let $f:[a, b] \rightarrow \mathbb{R}$. If $f:[a, b] \rightarrow \mathbb{R}$. $f$ is said to be Gauge integrable over $[a, b]$ if there exists $A \in \mathbb{R}$ such that for every $\varepsilon>0$ there exists a Gauge $\gamma$ on $[a, b]$ such that $|S(f, D)-A|<\varepsilon$ whenever $D$ is a $\gamma-f i n e$ tagged partition of $[a, b]$. The number $A$ is called the Gauge integral of $f$ over $I=[a, b]$ and is

2020 Mathematics Subject Classification. 40F06, 40G01.

Keywords. Gauge Integration, statistical convergence, measurable function, summability theory, cardinality. 
denoted by $\int_{a}^{b} f$ or $\int_{I} f$; when we encounter integrals depending upon parameters, it is also convenient to write $\int_{a}^{b} f(t)$ or $\int_{I} f(t)$.

Throughout this paper we shall use the notion of bounded variation which is as follows: Let $f$ be a function on $[a, b]$. Given a partition $P=\left\{\left[x_{k-1}, x_{k}\right]\right\}$ of $[a, b]$, the variation of $f$ with respect to $P$ is

$$
V(f, P)=\sum_{k}\left|f\left(x_{k}\right)-f\left(x_{k-1}\right)\right|,
$$

and the variation of $f$ over $[a, b]$ is

$$
V_{a}^{b} f=\sup _{P} V(f, P),
$$

where the supremum is taken over all partitions $P$ of $[a, b]$. If $V_{a}^{b} f$ is finite, then $f$ is said to be of bounded variation on $[a, b]$. The set of all such functions is denoted by $B V([a, b])$.

On the other hand, in 1951 Fast [2] introduced an extension the concept of sequential limit to statistically convergence which as follows:

Definition 1.2. If $\mathbb{N}$ denotes the set of natural numbers and $K \subset \mathbb{N}$, then $K(m, n)$ denotes the cardinality of the set $K \cap[m, n]$. The upper and lower natural density of the subset $K$ is defined by

$$
\bar{d}(K)=\lim _{n \rightarrow \infty} \sup \frac{K(1, n)}{n} \text { and } \underline{d}(K)=\lim _{n \rightarrow \infty} \inf \frac{K(1, n)}{n} .
$$

If $\bar{d}(K)=\underline{d}(K)$, then we say that the natural density of $K$ exists and it is denoted simply by $d(K)$. Clearly, $d(K)=\lim _{n \rightarrow \infty} \frac{K(1, n)}{n}$. A sequence $x=\left(x_{k}\right)$ of real numbers is said to be statistically convergent to $L$ if for arbitrary $\varepsilon>0$, the set $K(\varepsilon)=\left\{k \in \mathbb{N}:\left|x_{k}-L\right| \geq \varepsilon\right\}$ has natural density zero. In this case, we will denote statistically convergence as $s t-\lim x_{k}$.

Following Fast's definition Schoenberg in [10] presented a bridge of this concept to summability theory. Recently, statistical convergence has been one of the most active areas in summability theory thanks to Fridy's presentation in [3] and many other papers were studied in this area (see [7], [8]). Afterward, strongly summable single valued functions were studied by Borwein in [1]. Following Borwein's work Nuray [6] extended his notion via $\lambda$-strongly summability and $\lambda$-statistically convergent functions by taking nonnegative real-valued Lebesgue measurable function on $(1, \infty)$. Prior to present Nuray's notions, let us note that the following definition.

Definition 1.3. [6] Let $\lambda=\left(\lambda_{n}\right)$ be non-decreasing sequence of positive numbers tending to $\infty$ such that $\lambda_{n+1} \leq \lambda_{n}+1, \lambda_{1}=1$. $\Delta$ denote the set of all such sequences. For a sequence $x=\left(x_{n}\right)$ the generalized de la Vallée Poussin mean is defined by

$$
t_{n}(x)=\frac{1}{n} \sum_{k \in I_{n}} x_{k}
$$

where $I_{n}=\left[n-\lambda_{n}+1, n\right]$.

Definition 1.4. [6] Let $\lambda \in \Delta$ and $f(\psi)$ be a real valued function which is measurable in the Lebesgue sense in the interval $(1, \infty)$, if

$$
\lim _{n \rightarrow \infty} \frac{1}{\lambda_{n}} \int_{n-\lambda_{n}+1}^{n}|f(\psi)-L| d \psi=0,
$$


then we say that the function $f(\psi)$ is $\lambda$-strongly summable to $L$. In this case we write $[W, \lambda]-\lim f(\psi)=L$ or $f(\psi) \rightarrow L[W, \lambda]$. If we take $\lambda_{n}=n$, then $[W, \lambda]$ reduced to $[W]$, the space of all all strongly double summable functions.

Definition 1.5. [6] Let $\lambda \in \Delta$ and $f(\psi)$ be a real-valued function which is measurable on $(1, \infty)$, if for every $\varepsilon>0$,

$$
\lim _{n \rightarrow \infty} \frac{1}{\lambda_{n}}\left|\left\{\psi \in I_{n}:|f(\psi)-L| \geq \varepsilon\right\}\right|=0,
$$

then we say that the function $f(\psi)$ is $\lambda$-statistically convergent to $L$, where the vertical bars indicate the Lebesgue measurable of the enclosed set. The space of all statistical convergence functions will be denoted by $\left(S_{f}, \lambda\right)$. In this case, we write $\left[S_{f}, \lambda\right]-\lim f(\psi)=$ $L$ or $f(\psi) \rightarrow L\left[S_{f}, \lambda\right]$.

The following is an example of such convergence.

Example 1.6. Let us consider a function $f(\psi)$ which is defined by

$$
f(\psi)=\left\{\begin{array}{l}
\psi, n-\frac{1}{\log \lambda_{n}}+\frac{1}{\lambda_{n}}+1 \leq \psi \leq n \\
0, \text { otherwise }
\end{array}\right.
$$

for every $\varepsilon>0$,

$$
\lim _{n \rightarrow \infty} \frac{1}{\lambda_{n}}\left|\left\{\psi \in I_{n}:|f(\psi)-0| \geq \varepsilon\right\}\right|=\lim _{n \rightarrow \infty} \frac{\frac{1}{\log \lambda_{n}}+\frac{1}{\lambda_{n}}}{\lambda_{n}}=0,
$$

i.e., $\left[S_{f}, \lambda\right]-\lim f(\psi)=0$.

In addition to these definitions, please note the following theorem in [6].

Theorem 1.7. [6] Let $\lambda \in \Delta$ and $f(\psi)$ be a real valued function which is measurable in the Lebesgue sense in the interval $(1, \infty)$, then $[W, \lambda] \subset\left[S_{f}, \lambda\right]$ and the inclusion is proper.

In 2019, Savas and Patterson in [9] introduced the new concept of strongly Cesáro type summability theory by considering Gauge integral and the following definition:

Definition 1.8. [9] Let us consider $\delta: I_{i}=\left(t_{i}-\delta\left(t_{i}\right), t_{i}+\delta\left(t_{i}\right)\right] \rightarrow(0, \infty)$ is a positive function, and $[a, b]=\cup I_{i}$ with $-\infty<a<b<\infty$. We define an open interval valued function on $I$ by setting $\bar{\gamma}=\bar{\gamma}\left(t_{i}\right)=\left(t_{i}-\delta\left(t_{i}\right), t_{i}+\delta\left(t_{i}\right)\right)$. If $J_{i}=\left[i-\lambda_{i}+1, i\right]$, we can write $t_{i} \in J_{i} \subset \bar{\gamma}\left(t_{i}\right)$ instad of $t_{i}-\delta\left(t_{i}\right)<i-\lambda_{i}+1 \leq t_{i} \leq i<t_{i}+\delta\left(t_{i}\right)$. Let $\bar{\gamma}=\bar{\gamma}\left(t_{i}\right) \in \Delta_{G}$, and let $f(\psi)$ be a real valued function which is measurable Gauge sense in the interval $(1, \infty)$. Provided that $\int f(\psi)$ and $\int|f(\psi)|$ exist in the gauge sense and

$$
\lim _{t_{i} \rightarrow \infty} \frac{1}{\xi\left(t_{i}\right)} \int_{t_{i}-\delta\left(t_{i}\right)}^{t_{i}+\delta\left(t_{i}\right)}|f(\psi)-L| d \psi=0
$$

where $\xi\left(t_{i}\right)=\left(t_{i}+\delta\left(t_{i}\right)\right)-\left(t_{i}-\delta\left(t_{i}\right)\right)=2 \delta\left(t_{i}\right)$, then we say that the function $f(\psi)$ is $\bar{\gamma}$-strongly summable to $L$ with respect Gauge. In this case, we write $[G, \bar{\gamma}]-\lim f(\psi)=L$ or $f(\psi) \rightarrow L[G, \bar{\gamma}]$.

Using the definitions above, Savas and Patterson also established the following theorem which grants us a connection between strongly summability in the Lebesgue sense and in the Gauge sense.

Theorem 1.9. [9] Let $\lambda=\left(\lambda_{n}\right) \in \Delta, \bar{\gamma}=\bar{\gamma}\left(t_{i}\right) \in \Delta_{G}, I_{i}=\left[t_{i}-\delta\left(t_{i}\right), t_{i}+\delta\left(t_{i}\right)\right]$ and $[a, b]=\cup I_{i}$ with $-\infty<a<b<\infty$, and $f(\psi)$ be a real valued function in the Gauge sense in the interval $(1, \infty)$, then

(1) $[W, \lambda] \subset[G, \bar{\gamma}]$ 
(2) If $f(\psi)$ is bounded variation and $f$ is $\bar{\gamma}$-strongly summable to $L$ with respect to Gauge sense over every measurable subset of $\left[t_{i}-\delta\left(t_{i}\right), t_{i}+\delta\left(t_{i}\right)\right]$ (i.e., if $C_{E} f$ is Gauge integrable over $\left[t_{i}-\delta\left(t_{i}\right), t_{i}+\delta\left(t_{i}\right)\right]$ ) for every measurable $\left.E \subset t_{i}-\delta\left(t_{i}\right), t_{i}+\delta\left(t_{i}\right)\right)$, then $f$ is $[W]-\lim f(\psi)=L$.

\section{MAin Results}

We begin this section with the following new definition.

Definition 2.1. Let $\lambda \in \Delta$ and $f(\psi)$ be a real-valued function in the interval $(1, \infty)$, for every $\varepsilon>0$, let $A=\left\{\psi \in I_{n}:|f(\psi)-L| \geq \varepsilon\right\},\left\{A_{i}: i \in \mathbb{N}\right\}$ be a countable partition of $A$, and $\alpha_{i}=\sup \left\{\psi \in A_{i}\right\}$. Provided that

$$
\lim _{n \rightarrow \infty} \frac{1}{\alpha_{n}}\left|\left\{\psi \leq \alpha_{i}:|f(\psi)-L| \geq \varepsilon\right\}\right|=0,
$$

where the vertical bars indicate the Lebesgue measure of the enclosed set, then we say $f(\psi)$ is statistically convergent to $L$ via cardinality. In this case, we write $S_{f}^{*}-\lim f(\psi)=L$ or $f(\psi) \rightarrow L\left[S_{f}^{*}\right]$. The class of the $\lambda$-statistically convergent to $L$ via cardinality is denoted by $\left[S_{f}^{*}\right]$.

This following are examples of a measurable and non-measurable functions, respectively that satisfy Definition 2.1.

Example 2.2. $f(\psi)$ be a real-valued function which is measurable on $(1, \infty)$. Define by

$$
f(\psi)= \begin{cases}1 & \text { if } \psi \text { is a square } /\{1\} \\ 0 & \text { if } \psi \in(1, \infty) / \psi \text { is not a square. }\end{cases}
$$

Example 2.3. Let $S$ a non-measurable subset of $(1, \infty)$. Define a function $f(\psi)$ by

$$
f(\psi)= \begin{cases}1 & \text { if } \psi \in S \cup(\psi \text { is an even square }), \\ 0 & \text { if } \psi \in S \cup(\psi \text { is an odd square }), \\ 0 & \text { if otherwise. }\end{cases}
$$

Let us consider the following inclusion theorems.

Theorem 2.4. If $\lim _{n \rightarrow \infty} \inf \frac{\lambda_{n}}{\alpha_{n}}>0$ and $\frac{\lambda_{n}}{\alpha_{n}}=O(1)$, then $\left[S_{f}, \lambda\right] \subseteq\left[S_{f}^{*}\right]$.

Proof. Let $\varepsilon>0$ and $\left[S_{f}, \lambda\right]-\lim f(\psi)=L$. We write

$$
\left\{\psi \leq \alpha_{i}:|f(\psi)-L| \geq \varepsilon\right\} \supset\left\{\psi \in I_{n}:|f(\psi)-L| \geq \varepsilon\right\} .
$$

Therefore,

$$
\begin{aligned}
\frac{1}{\alpha_{n}}\left|\left\{\psi \leq \alpha_{i}:|f(\psi)-L| \geq \varepsilon\right\}\right| & \geq \frac{1}{\alpha_{n}}\left|\left\{\psi \in I_{n}:|f(\psi)-L| \geq \varepsilon\right\}\right| \\
& \geq \frac{\lambda_{n}}{\alpha_{n}} \cdot \frac{1}{\lambda_{n}}\left|\left\{\psi \in I_{n}:|f(\psi)-L| \geq \varepsilon\right\}\right| .
\end{aligned}
$$

Hence by using $\lim _{n \rightarrow \infty} \inf \frac{\lambda_{n}}{\alpha_{n}}>0$ and taking the limit $n \rightarrow \infty$ we get $f(\psi) \rightarrow L\left[S_{f}, \lambda\right]$ implies $f(\psi) \rightarrow L\left[S_{f}^{*}\right]$.

Theorem 2.5. $[W, \lambda] \subset\left[S_{f}^{*}\right]$ and for the condition $\lim _{n \rightarrow \infty} \inf \frac{\alpha_{n}}{\lambda_{n}}>1$, the inclusion is proper. 
Proof. Let $\varepsilon>0$ and $[W, \lambda]-\lim f(\psi)=L$. We write

$$
\int_{\psi \in I_{n}}|f(\psi)-L| d \psi=\int_{\left\{\psi: \psi \leq \alpha_{i}\right\}}|f(\psi)-L| d \psi \geq \varepsilon\left\{\psi \leq \alpha_{i}:|f(\psi)-L| \geq \varepsilon\right\} .
$$

Therefore, $[W, \lambda]-\lim f(\psi)=L$ implies $S_{f}^{*}-\lim f(\psi)=L$. Let us consider the following function

$$
f(\psi)= \begin{cases}\psi, & n-\ln \left(\lambda_{n}\right)+1 \leq \psi \leq n \\ 0 & \text { otherwise }\end{cases}
$$

$f(\psi)$ is not bounded function, for every $\varepsilon>0$,

$$
\begin{aligned}
& \lim _{n \rightarrow \infty} \frac{1}{\alpha_{n}}\left|\left\{\psi \leq \alpha_{i}:|f(\psi)-L| \geq \varepsilon\right\}\right| \\
\geq & \lim _{n \rightarrow \infty} \frac{1}{\alpha_{n}} \frac{\alpha_{n}}{\lambda_{n}}\left|\left\{\psi \leq \alpha_{i}:|f(\psi)-L| \geq \varepsilon\right\}\right| \\
= & \lim _{n \rightarrow \infty} \frac{\ln \left(\lambda_{n}\right)}{\lambda_{n}}=0,
\end{aligned}
$$

i.e., $S_{f}^{*}-\lim f(\psi)=0$. However,

$$
\lim _{n \rightarrow \infty} \frac{1}{\lambda_{n}} \int_{n-\lambda_{n}+1}^{n}|f(\psi)-0| d \psi=\infty
$$

i.e., $f(\psi) \nrightarrow L[W, \lambda]$. Hence, the inclusion is proper.

Theorem 2.6. $[G, \bar{\gamma}] \varsubsetneqq\left[S_{f}^{*}\right]$.

Proof. Suppose that $\varepsilon>0,[G, \bar{\gamma}]-\lim f(\psi)=L$. Therefore, we can obtain the following

$$
\begin{aligned}
\int_{\psi \in \bar{\gamma}\left(t_{i}\right)}|f(\psi)-L| d \psi & \geq \int_{\left\{\psi \in \bar{\gamma}\left(t_{i}\right):|f(\psi)-L| \geq \varepsilon\right\}}|f(\psi)-L| d \psi \\
& \geq \varepsilon\left|\left\{\psi \in \bar{\gamma}\left(t_{i}\right):|f(\psi)-L| \geq \varepsilon\right\}\right| \\
& \geq \varepsilon\left|\left\{\psi \leq \alpha_{i}:|f(\psi)-L| \geq \varepsilon\right\}\right|
\end{aligned}
$$

which implies that $f(\psi) \nrightarrow L\left[S_{f}, \lambda\right]$.

Theorem 2.7. If $\lim _{n \rightarrow \infty} \inf \frac{\bar{\gamma}\left(t_{i}\right)}{\alpha_{n}}>0$ and $f(\psi)$ is a bounded variation, then $\left[S_{f}^{*}\right] \subseteq[G, \bar{\gamma}]$.

Proof. Suppose that $\left[S_{f}^{*}\right]-\lim f(\psi)=L$ and since $f(\psi)$ be a bounded variation, $f(\psi)$ will be a bounded function, and we say that $|f(\psi)-L| \leq M$ for all $\psi$. Given $\varepsilon>0$, we have that

$$
\begin{aligned}
\frac{1}{\bar{\gamma}\left(t_{i}\right)} \int_{\psi \in \bar{\gamma}\left(t_{i}\right)}|f(\psi)-L| d \psi= & \frac{1}{\bar{\gamma}\left(t_{i}\right)} \int_{\left\{\psi \in \bar{\gamma}\left(t_{i}\right):|f(\psi)-L| \geq \varepsilon\right\}}|f(\psi)-L| d \psi \\
& +\frac{1}{\bar{\gamma}\left(t_{i}\right)} \int_{\left\{\psi \in \bar{\gamma}\left(t_{i}\right):|f(\psi)-L|<\varepsilon\right\}}|f(\psi)-L| d x \\
\leq & \frac{M}{\bar{\gamma}\left(t_{i}\right)}\left|\left\{\psi \in \bar{\gamma}\left(t_{i}\right):|f(\psi)-0| \geq \varepsilon\right\}\right|+\varepsilon \\
\leq & \frac{M}{\bar{\gamma}\left(t_{i}\right)}\left|\left\{\psi \leq \alpha_{i}:|f(\psi)-L| \geq \varepsilon\right\}\right|+\varepsilon \\
\leq & \frac{\bar{\gamma}\left(t_{i}\right)}{\alpha_{n}} \frac{M}{\bar{\gamma}\left(t_{i}\right)}\left|\left\{\psi \leq \alpha_{i}:|f(\psi)-L| \geq \varepsilon\right\}\right|+\varepsilon
\end{aligned}
$$


Hence, $[G, \bar{\gamma}]-\lim f(\psi)=L$.

Acknowledgement: The first author is thankful to TUBITAK for almost two year Visiting Scientist at University of North Florida, Jacksonville, U.S.A. where this work was done during 2017-2019.

\section{REFERENCES}

[1] D. Borwein, Linear Functionals with Strongly Cesáro Summability, Journal of London Mat. Soc., 40 (1965), 628-634.

[2] H. Fast, Sur la Convergence Statistique, Colloquium Mathematicum, 2 (1951), 241-244.

[3] J. A. Fridy, On Statistically Convergence, Analysis, 5 (1985), 301-313.

[4] R. Henstock, Definitions of Riemann Type of Variational Integral, Proc. London Math. Soc., 11 (1961), 402-418.

[5] J. Kurzweil, Generalized Ordinary Differential Equations and Continuous Dependence on a Parameter, Czech. Math. J., 82 (1957), 418-449.

[6] F. Nuray, $\lambda$-Strongly Summable and $\lambda$-Statistically Convergent Functions, Iranian Journal of Sci. and Tech., 34 (4) (2010), 335-338.

[7] K. Raj, A. Choudhary and C. Sharma, Almost strongly Orlicz double sequence spaces of regular matrices and their applications to statistical convergence, Asian-Eur. J. Math., Vol.11 No.5 (2018), 1850073, (14pages).doi.org/10.1142/S1793557118500730.

[8] K. Raj and S. Jamwal, On some generalized statistical convergent sequence spaces, Kuwait J. Sci., 42 (3) (2015), 86-104.

[9] R. Savas and R. F. Patterson, Gauge Strongly Summability for Measurable Functions, Carpathian Journal of Mathematics, (accepted-preprint)

[10] I. J. Schoenberg, The Integrability of Certain Functions and Related Summability Theory, Am. Math. Month., 66 (1959), 361-375.

[11] C. Swartz, Introduction to Gauge Integrals, World Scientific Publishing Co., 1938.

RABİA SAVAŞ: rabiasavass@hotmail.com

Department of Mathematics, Sakarya University, Sakarya, Turkey

Richard F. PATterson: rpatters@unf.edu

Department of Mathematics and Statistics, University of North Florida, Jacksonville, Florida

Received 04/04/2020; Revised 18/11/2020 